\title{
Estudo Prospectivo para Avaliação de Tecnologias de Reatores Químicos
}

\section{Prospective Study for Evaluation of Chemical Reactor Technologies}

\author{
Wilton Lima Silva Saturnino ${ }^{1}$ \\ Sidinei Kleber Silva ${ }^{2}$ \\ ${ }^{1}$ Universidade Federal de Campina Grande, Campina Grande, PB, Brasil \\ ${ }^{2}$ Universidade Federal da Paraíba, João Pessoa, PB, Brasil
}

\begin{abstract}
Resumo
A prospecção tecnológica com base em bancos de patentes consiste em uma forma de se obter uma tendência na qual uma tecnologia pode se desenvolver buscando respostas relevantes para uma possível tomada de decisão em áreas de gerenciamento e negócios. Desta forma, este trabalho teve como foco obter informações contidas em banco de patentes e verificar, no estado da técnica, projeções ou tendência atuais para tecnologias de reatores químicos. Foram realizadas buscas nos principais bancos de patentes gratuitos, utilizando-se para isso uma ferramenta de busca on-line de licença gratuita da tecnologia Octimine ${ }^{\circledR}$. Os resultados mostram que as engenharias mecânica, química e elétrica apresentam os maiores números de depósitos, sendo as tecnologias de refrigeração e troca térmica, reator nuclear e um método de manufatura de um micro reator com canal de fluxo as tecnologias protegidas de maior representatividade, de maneira que a Europa e, especificamente, a Alemanha é onde mais se depositam patentes.
\end{abstract}

Palavras-Chave: Prospecção Tecnológica. Patentes. Reatores Químicos.

\begin{abstract}
Technology-based patent-based prospecting is one way to obtain a trend in which a technology can evolve by seeking relevant answers to a possible decision-making in management and business areas. In this way, the following work focused on obtaining information contained in a patent bank and verifying in the state of the art, current projections or trends for chemical reactor technologies. Searches were carried out at the main free patent banks, using an online search tool for free license of Octimine ${ }^{\circledast}$ technology. The results show that mechanical, chemical and electrical engineering have a greater number of deposits, with refrigeration and exchange technologies thermal reactor and a manufacturing method of a micro-reactor with flow channel as the most representative protected technologies, so that Europe and Germany are the countries that most deposit patents.
\end{abstract}

Keywords: Technological Prospecting. Patents. Chemical Reactors.

Área tecnológica: Propriedade Intelectual.

\section{Introdução}

A prospecção tecnológica de forma genérica pode ser definida como uma ferramenta sistemática que auxilia a sociedade na tomada de decisão, buscando uma visão viável para um investimento futuro com base em tendências estimuladas pelas condições atuais da ciência, tecnologia e cultura. É importante salientar que a prospecção é uma ferramenta básica e consistente que orienta a tomada de decisão na sociedade moderna e não consiste em prever o 
futuro, mas sim criar uma estratégia de decisão no presente com o objetivo de obter melhores vantagens no futuro (MAYERHOFF, 2008).

Este trabalho busca avaliar a tendência na qual os estudos na área de reatores químicos se desenvolvem com base na busca no estado da técnica, utilizando-se para isso a prospecção tecnológica em bancos de dados com abrangência mundial e considerando-se a importância dessas bases para a propriedade intelectual.

Assim, coletaram-se dados sobre os pesquisadores, as empresas envolvidas, países que mais publicam na área e as tecnologias protegidas por seus inventores. Para isso, alguns critérios de busca específicos para o tema de interesse foram utilizados, e os resultados obtidos numa lista de patentes selecionadas foram analisados a fim de filtrar as informações de melhor interesse, com o objetivo de compreender a tendência para a qual os dados apontam ((SPEZIALI; SINISTERRA, 2012)

Alguns trabalhos disponíveis na literatura, como o de GOIS et al. (2012), no qual foi feita uma avaliação de tecnologias em reatores para produção de biodiesel a partir da análise dos pedidos de patentes, e a dissertação de Cruz (2015) sobre a aplicação de um novo reator de fluxo oscilatório no controle da qualidade e tamanho dos cristais de paracetamol, serviram de inspiração para o desenvolvimento deste trabalho devido à importância desse equipamento para a engenharia química e para o setor industrial.

\section{Metodologia}

A metodologia deste trabalho está voltada ao uso da ferramenta computacional Octimine ${ }^{\circledR}$, na qual, fazendo-se o uso de palavras-chave, é possível obter resultados de grupos de patentes em diversos países, tipos de análises gráficas e diagramas com vários tipos de aplicações.

Este software não é a única ferramenta de busca a fazer essas análises, mas enquanto a maioria das plataformas possui uma licença de uso paga, como por exemplo o Questel Orbit ${ }^{\circledR}$ e o Dialog ${ }^{\circledR}$, a Octimine ${ }^{\circledR}$ disponibiliza uma licença gratuita com certas limitações, mas ainda assim apresenta resultados abrangentes e muito intuitivos. Para realizar a prospecção tecnológica foi aplicada uma estratégia de busca usando as palavras chaves "Reactor", "Chemical", Reactor*, Convert*, Chemical Transformator, Chemical Transformator*. A pesquisa foi feita para melhor averiguação usando-se os termos separadamente e depois combinados, aplicando-se o operador lógico "AND".

A busca no estado da técnica por patentes não é nada fácil, e é preciso muito tempo combinando palavras-chave, operadores booleanos, truncamentos entre outras formas de filtragem da informação, porém o algoritmo desta ferramenta opera semanticamente para trabalhar com o número de patentes, palavras-chaves ou copiar textos em inglês, de maneira que são realizadas as combinações necessárias.

O Octamine ${ }^{\circledR}$ reconhece a escrita e seu contexto, sendo identificadas as palavras-chave mais pertinentes. Ao contrário das pesquisas booleanas tradicionais, não é preciso combinar todas as palavras e recurso de filtros que geralmente são utilizados em bancos de patentes.

Após a inserção das palavras-chaves e a configuração dos operadores lógicos, solicita-se a busca na plataforma Octamine ${ }^{\circledR} e$, após alguns segundos, é possível observar os resultados na tela. A presente resposta foi gerada com uma lista com mais de 1.000 patentes similares aos 
termos pesquisados, mostrando as 25 patentes mais relevantes por página, todas disponíveis para baixar nos formatos PDF, CSV ou Excel.

Para obter a lista de resultados basta clicar na ferramenta de exportação de arquivos, podendo selecionar todos os resultados ou individualmente. Os gráficos plotados descrevem análises para os resultados das mais de mil patentes analisadas, no entanto cerca de vinte e cinco a trinta patentes mais relevantes com a procura estarão representados nos gráficos visualizados.

\section{Resultados e Discussão}

Há vários escritórios nos quais podem ser depositadas as patentes de invenção. Contudo, a depender do banco de patentes, a abrangência territorial da patente poderá ser maior. O Gráfico 1 ilustra o número famílias de patentes versus autoridades, e é possível observar os bancos de patente onde foi feito o depósito pelo mundo, considerando-se as patentes mais relevantes ao assunto.

Nota-se que a WO (WIPO), Europa (EP) e a Alemanha (DE) têm um impacto muito relevante no assunto em relação aos demais bancos de patentes, o que faz todo sentido já que a Europa e, principalmente, a Alemanha investem pesado na educação e, com destaque, nas áreas de engenharia e ciência da computação. O Brasil, infelizmente, não aparece ranqueado, já que existem poucas patentes relevantes ao assunto para que o país seja representado nesta lista.

É preciso mencionar que, nos resultados gerados pelo Octimine ${ }^{\circledR}$, os gráficos e diagramas obtidos são, em sua grande maioria, interativos e revelam informações extras quando aproxima-se o ponteiro do mouse do computador por uma região específica, ou quando aciona-se um clique em alguma informação, e a busca se torna mais aprofundada.

No caso do Gráfico 1, acionar um clique sobre qualquer uma das barras em que são mostradas as autoridades, o número exato de famílias de patentes registrada é informado ao usuário do sistema. Desta forma, a autoridade WO recebeu 23 registros de famílias de patentes e, portanto, é o banco de patentes que aparece em primeiro lugar nesta lista.

Gráfico 1 - Número de família de patentes por autoridades

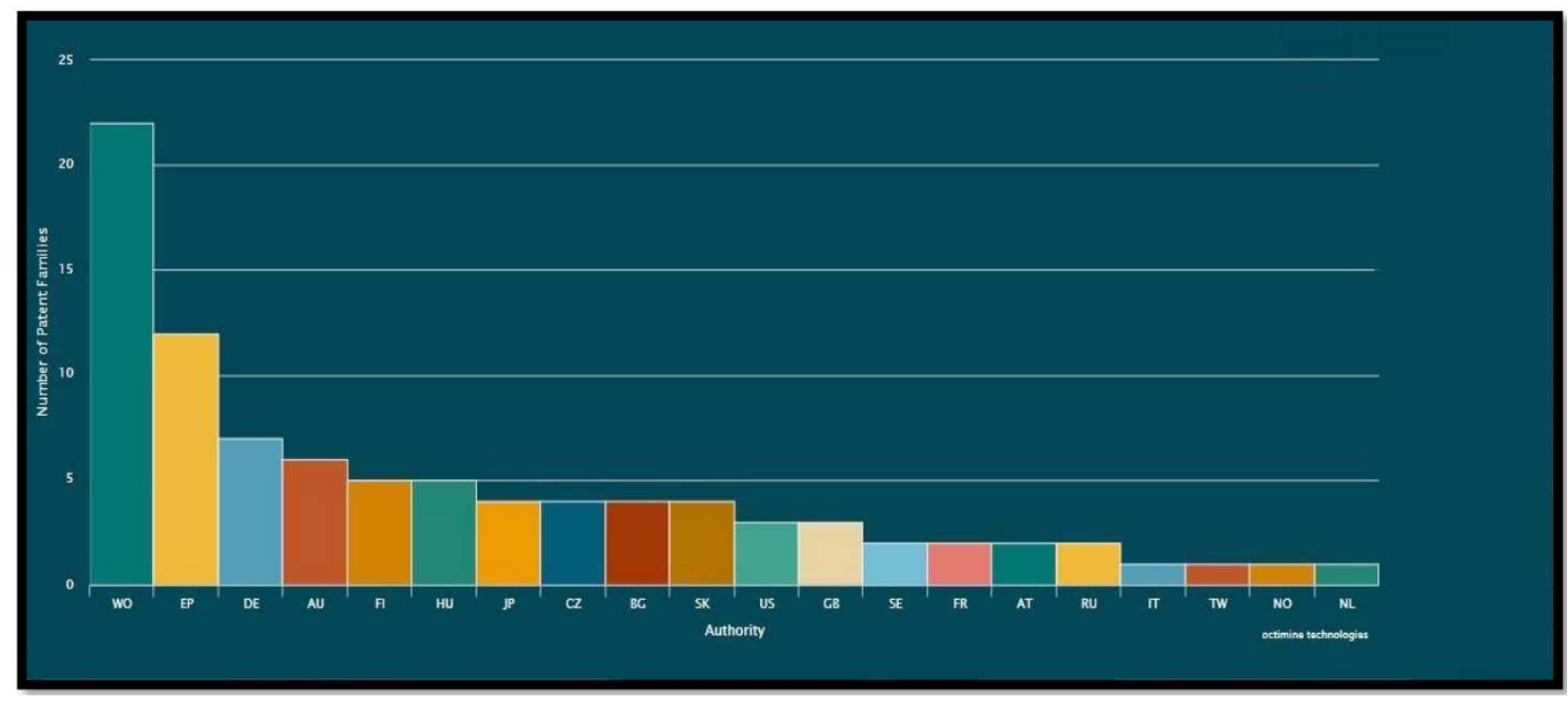

Fonte: Elaborado pelos autores deste artigo (2018) 


\subsection{Curva de Densidade de Similaridade}

É possível verificar a similaridade, ou seja, a concordâncias da lista de resultados com os termos usados na busca. No Gráfico 2 é apresentada a densidade de similaridade, onde são relacionadas as patentes mais significativas ou similares às palavras-chaves utilizadas, numa escala que vai de 0 a 1.000. Porém, neste gráfico a escala foi aproximada de 400 a 1.000 para melhorar a visualização dos resultados.

No primeiro ponto do ranking foi obtida a seguinte informação (gráfico interativo): Publication Number: FR2665645A1; Rank: 1; Similarity Index: 892. A invenção é o Method And Device For Heating And Cooling A Chemical Reactor do autor Christian Allemann, da empresa Sundgau Sarl Atel Const Elect.

É possível observar que é uma patente depositada na França com um código de tipo A1, e apresenta similaridade 892 com a busca realizada. Um dos critérios de seleção das tecnologias protegidas é justamente a similaridade com os resultados, pois representa a relevância do arquivo encontrado com as palavras-chave utilizadas na pesquisa de interesse. No decorrer deste trabalho alguns exemplos de tecnologia protegida serão demonstrados.

Gráfico 2 - Densidade de similaridade

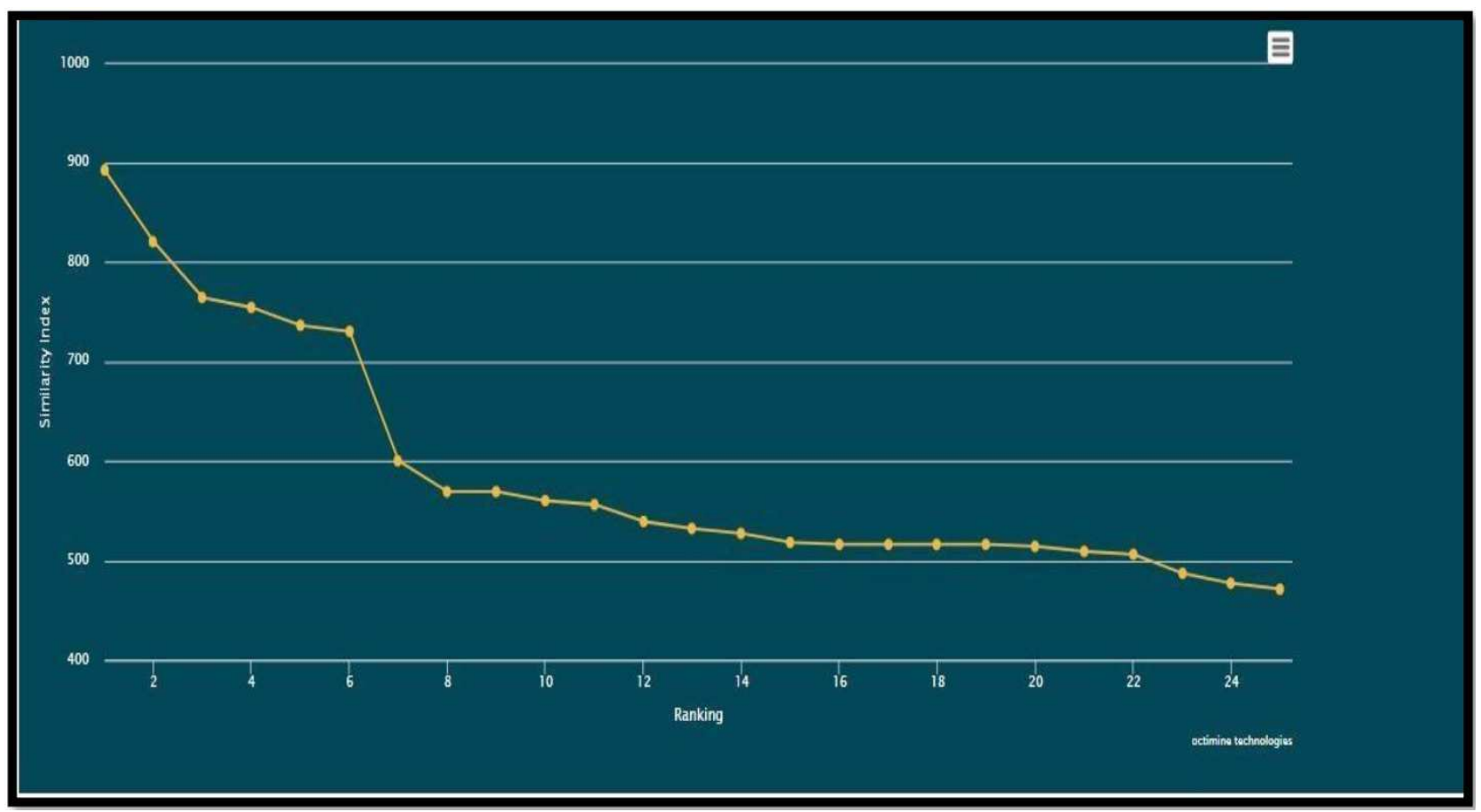

Fonte: Elaborado pelos autores deste artigo (2018)

\subsection{Campos de Tecnologia ao Longo do Tempo - Mapa de Calor}

A lista de resultados descreve datas e mais de trinta e cinco campos da tecnologia que podem ser estudados. O Gráfico 3 mostra um mapa de calor onde são analisados os campos da tecnologia mais aquecidos em um intervalo de tempo medido em anos. Observe que os espaços individuais são classificados por cores: quanto mais intensa a cor, maior será o número de patentes publicadas para aquela área. 
A partir destes dados observa-se o desenvolvimento dos campos tecnológicos ao longo do tempo, sendo que a intensidade na qual o tema gerou mais relevância para a época está representado pela cor cinzenta, que vai de 0 até 2 . Já os quadros vazios ou em azul indicam que não houve registros de depósitos de patentes suficientes para serem demonstrados neste gráfico.

O campo da tecnologia que mais ganhou destaque foi o da engenharia química, sendo que, pelos dados apurados, este campo ficou em alta por cinco temporadas, o que faz todo o sentido, já que este é um equipamento bastante explorado pelo profissional formado nessa área. Portanto, é um dos seus objetivos também o estudo e o aperfeiçoamento de tal equipamento.

Gráfico 3 - Campos da tecnologia ao longo do tempo

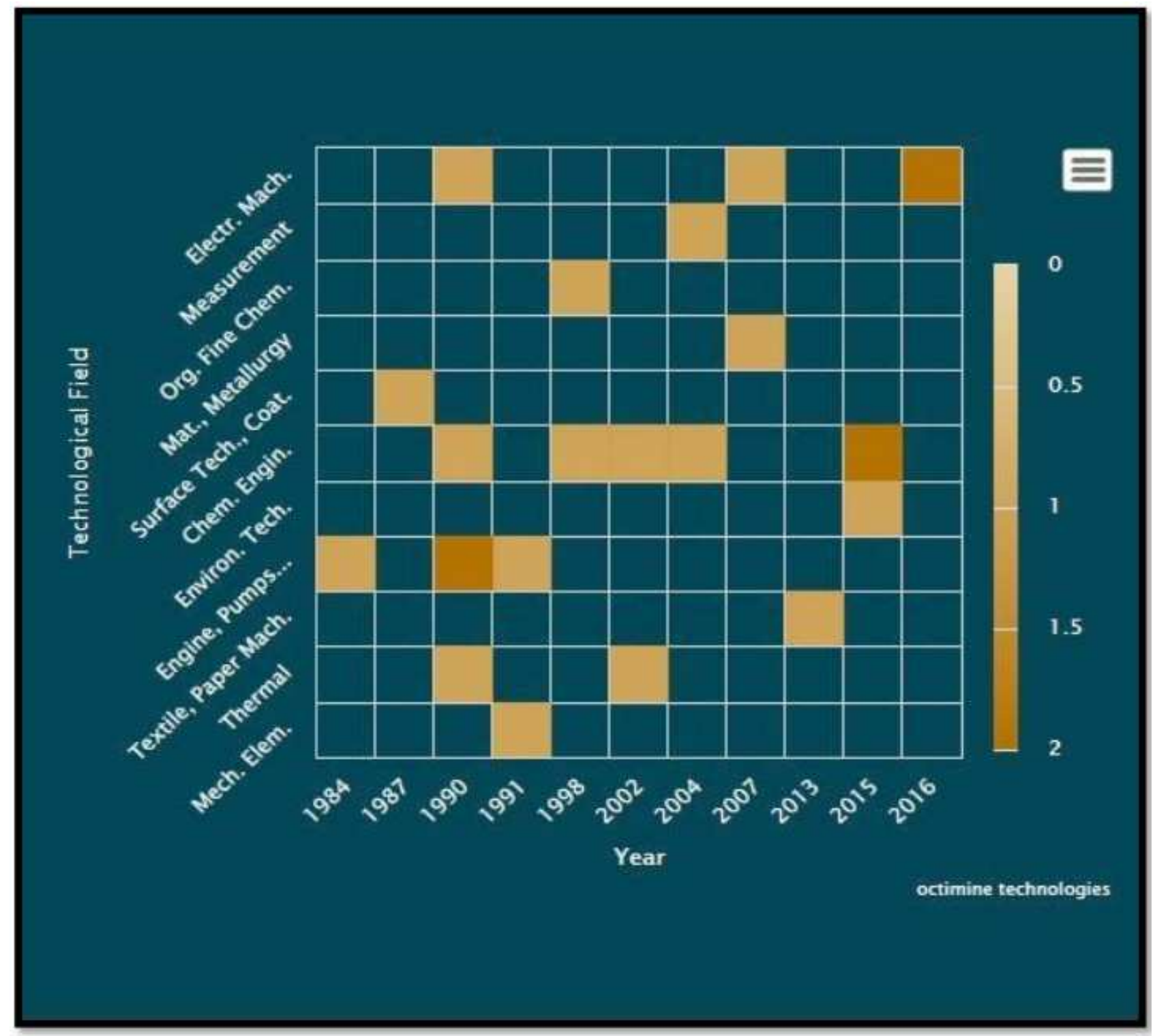

Fonte: Elaborado pelos autores deste artigo (2018)

Para este estudo a escala cronológica foi estabelecida entre os 1984 até 2016, logo é possível observar que, em um quadro contemporâneo, o campo para investimento em engenharia elétrica se apresenta bastante favorável, já que, em relação à busca, o desenvolvimento do maquinário elétrico, aparatos e redução de consumo energético estão em alta no quesito de publicações de patentes desde 2016. 


\subsection{Principais Depositantes}

Um gráfico estilo pizza pode representar bem a participação das empresas que têm como base as famílias de patentes atribuídas à lista de resultados selecionados. O Gráfico 4, principais depositantes do tipo $\mathrm{A}$, representa as ações dos cinco principais candidatos (empresas). Já o Gráfico 5, principais depositantes do tipo B, representa uma melhor visualização da distribuição dos depósitos realizados pelas empresas que são citadas como Others no Gráfico 4.

Gráfico 4 - Principais depositantes A

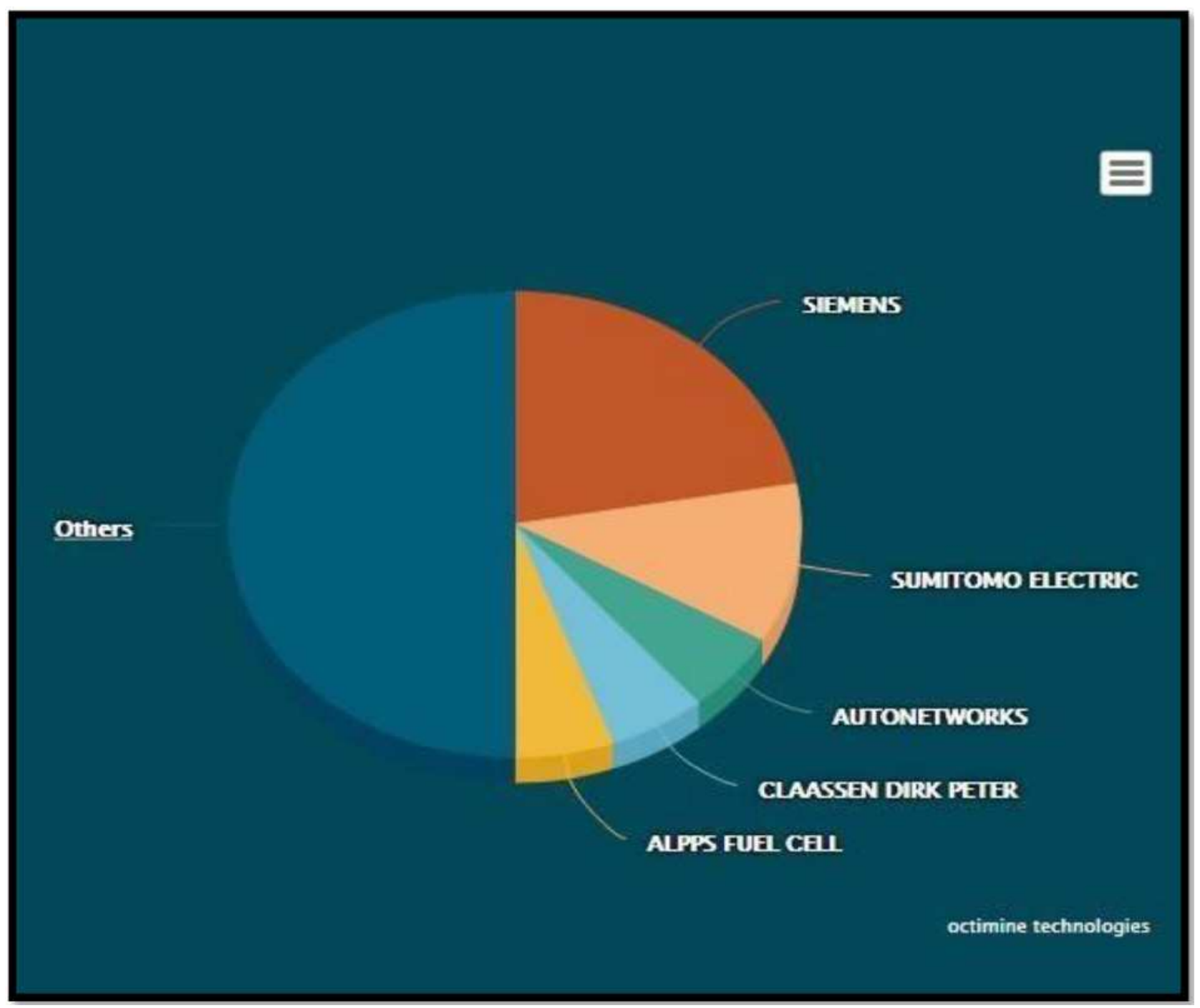

Fonte: Elaborado pelos autores deste artigo (2018)

Em geral, a lista de resultados confere cerca de 1.000 famílias de patentes, na qual o Gráfico 4 apresenta as empresas que mais investem em depósitos de patentes. Assim, destacaram-se as empresas Siemens e Sumitomo Eletric, que representam mais de 15 e 11 por cento, respectivamente, lembrando que este é um gráfico interativo e, por isso, seus valores numéricos não aparecem.

Como a porção do gráfico de pizza com o índice Others é muito grande, eles foram representados em um gráfico separadamente (Gráfico 5).

Gráfico 5 - Principais depositantes B 


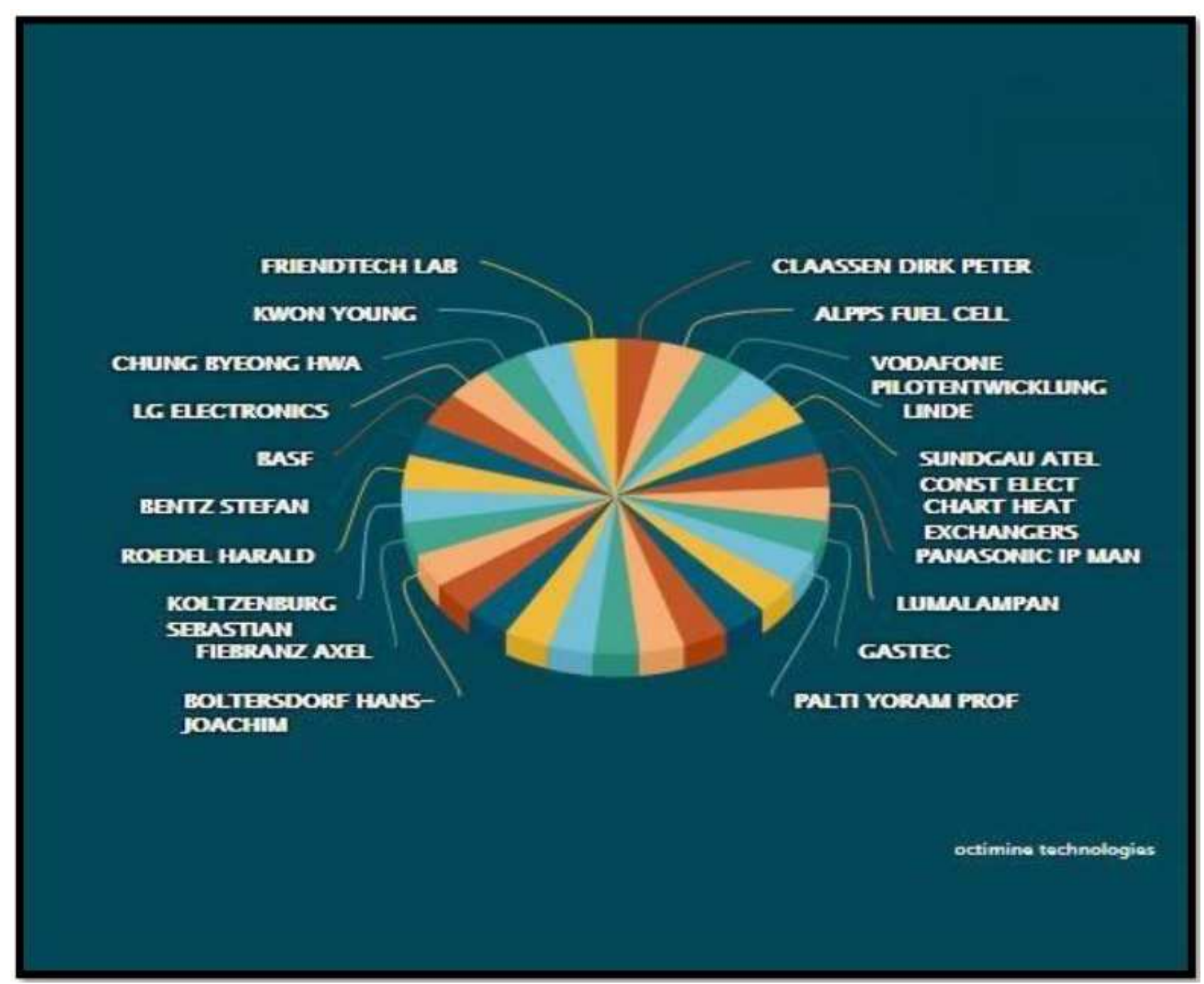

Fonte: Elaborado pelos autores deste artigo (2018)

Observou-se a participação de empresas especializadas e muito conhecidas mundialmente, com destaque para a LG Eletronics ${ }^{\circledR}$, conhecida empresa Sul-coreana, que começou com produtos de cosméticos e hoje está entre as maiores do setor eletrônico, principalmente, no desenvolvimento de tecnologias de smartphones e telas de imagem (KLEINA, 2017).

Outra empresa nesta lista é a empresa alemã BASF, uma das líderes no setor de indústria química, a qual iniciou sua história industrial no setor de corantes, e hoje, por exemplo, é uma das principais fornecedoras de vitaminas para nutrição humana e animal (BASF, 2018).

\subsection{Top Campos da Tecnologia}

As famílias de patentes podem ser distribuídas em 35 campos da tecnologia, determinados por Ulrich Schmoch e pelo Instituo Fraunhofer com base na lista de resultados obtidos na pesquisa (OCTIMINE, 2018). Tal classificação relaciona diferentes códigos IPC a campos da tecnologia de reatores químicos. O Top de campos tecnológicos "A" apresenta uma visão geral entre as áreas da ciência relacionadas com o resultado. Já o Top de campos tecnológicos "B" permite uma visão mais aprofundada em alguma das áreas. 
Gráfico 6 - Top campos da tecnologia

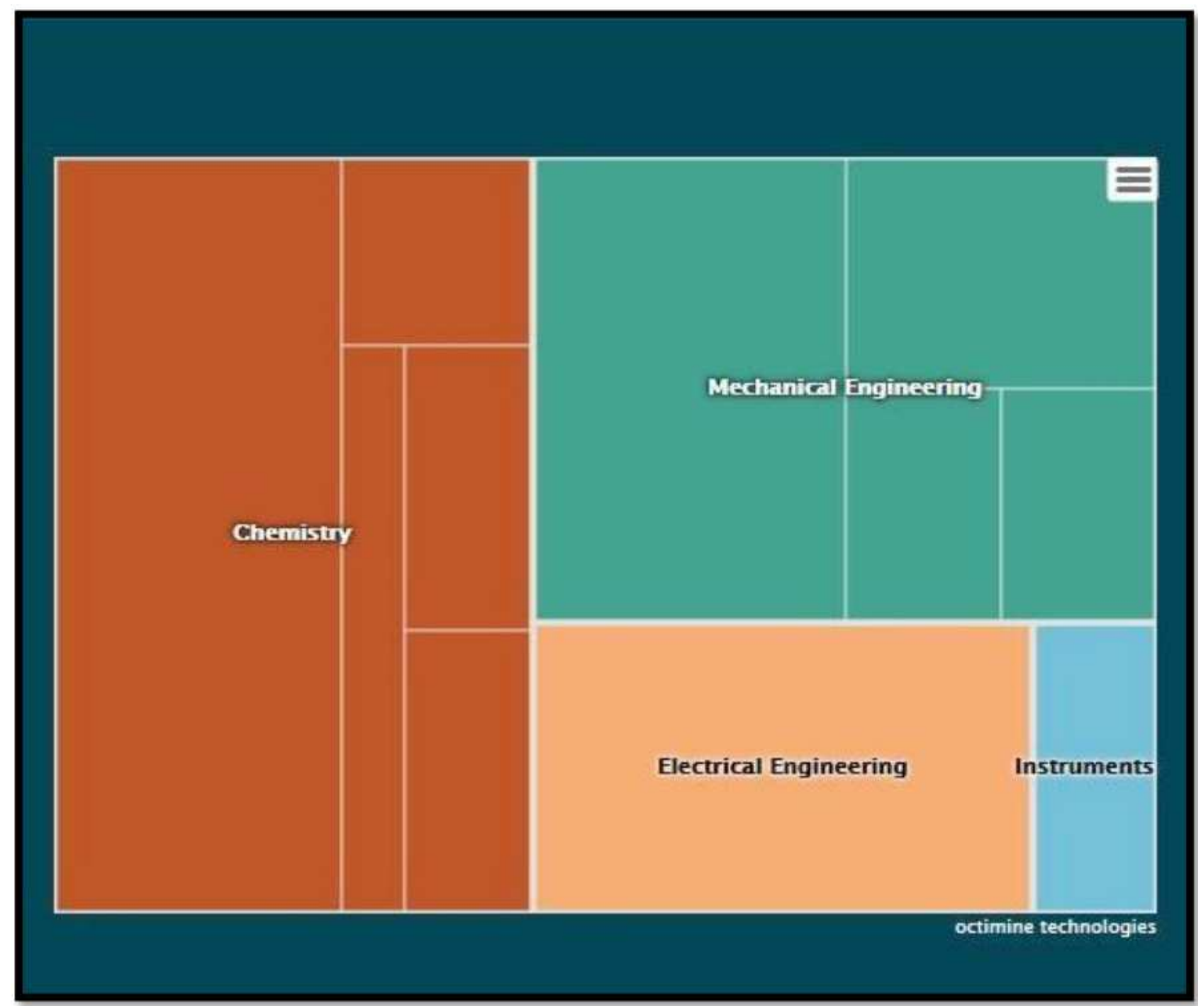

Fonte: Elaborado pelos autores deste artigo (2018)

Intuitivamente já era esperado que os campos da Engenharia Mecânica e da Química se destacariam com base na temática das palavras-chaves pesquisadas. O Gráfico 6, como o próprio nome sugere, passa a dimensão dos campos da ciência ao qual os reatores químicos estão envolvidos, sendo que estes reatores são "pulmões" da engenharia química que, desde as suas origens, tem uma relação muito forte com a engenharia mecânica.

Uma patente pode ser classificada em vários campos tecnológicos, logo as divisões mostradas do Gráfico 6 dizem respeito ao número total de diferentes patentes relacionadas aos seus correspondentes campos tecnológicos, sendo que esses valores podem ser acessados através de um clique no gráfico. Fazendo uma varredura mais aprofundada obtêm-se as subdivisões para o setor químico, como apresentado no Gráfico 7. 
Gráfico 7 - Top campos da tecnologia

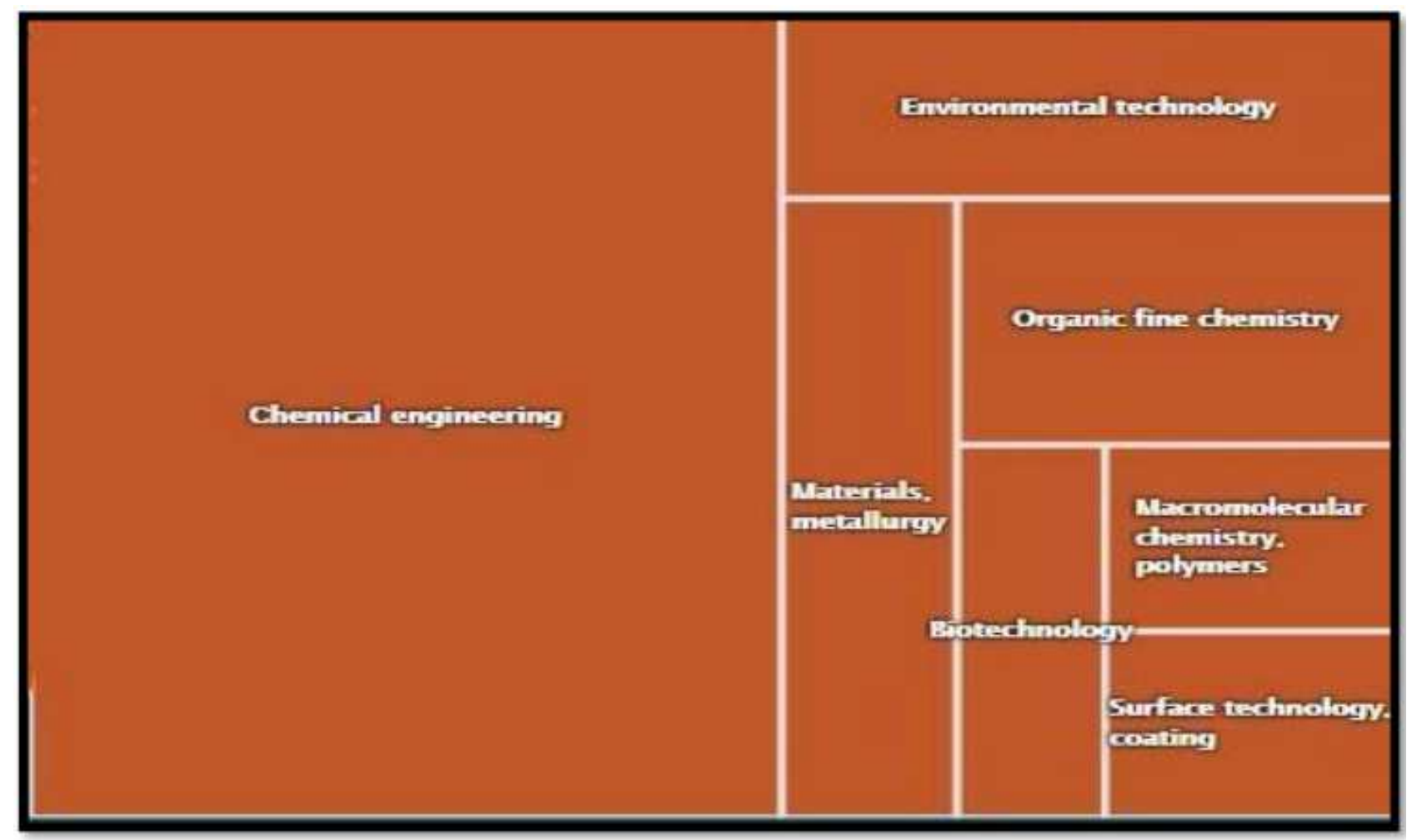

Fonte: Elaborado pelos autores deste artigo (2018)

Como pode ser observado, o campo da engenharia química apresenta 47,62\% e é expressivamente o mais abrangente para tecnologia de Reatores Químicos. Além disso, destaca-se a tecnologia em meio ambiente, com $14,29 \%$ das citações, uma vez que existe uma pressão muito grande dos governantes, mídias, organizações ambientais e da população pela redução da emissões, reaproveitamento e reciclagem de materiais poluentes ao meio ambiente.

\subsection{Tecnologias Protegidas}

Os resultados podem ser organizados numa lista onde são identificados os principais autores e as organizações responsáveis pelo depósito. Esta seleção está listada por ordem de similaridade com os termos da pesquisa. Logo, foi possível fazer uma busca nos bancos de patentes pelas tecnologias protegidas que mais se assemelham com a pesquisa.

A primeira tecnologia é a da empresa VODAFONE PILOTENTWICKLUNG, dos inventores BARTSCH ARMIN DR, HAUSINGER GUENTER DR, com a patente EP1224967A2, que se refere a reatores usados como, por exemplo, queimador catalítico, um permutador de calor ou similar.

Outra patente de grande valor científico é a invenção protegida pela empresa SIEMENS®, dos inventores SAUERMANN WALTER DIPL ING, MEIER WERNER DIPL ING. A invenção refere-se a um conjunto de montagem ou controle de combustível para um reator nuclear com uma caixa alongada, incluindo um interior com uma seção transversal poligonal e sendo definido por paredes laterais que se estendem paralelamente a um eixo longitudinal.

A tecnologia da empresa LONZA é atribuída aos inventores ROBERGE DOMINIQUE, GOTTSPONER MICHAEL, KOCKMANN NORBERT, EYHOLZER MARKUS. Seu número de proteção patentária é EP2429695A1. Esta tecnologia se refere a um método de fabricação de 
um reator e conjunto de reatores; esta invenção provê um método de fabricação de um reator alvo, especialmente um micro reator, atingindo uma vazão volumétrica alvo de um produto desejado por uma reação química que corre dentro do reator, alvo de uma pluralidade de reagentes ou instrutores.

\section{Considerações Finais}

Com o seguinte trabalho conclui-se que as tecnologias protegidas com maior similaridade demonstram tendência para o desenvolvimento em três diferentes áreas, sendo elas em: método de manufatura de reatores, desenvolvimento de micro reatores para processos catalíticos $e$ reatores nucleares, em nome das empresas LONZA ${ }^{\circledR}$, VODAFONE PILOTENTWICKLUNG ${ }^{\circledR} e$ SIEMENS ${ }^{\circledR}$, respectivamente. Essas são as tecnologias protegidas com maior similaridade com as palavras-chaves utilizadas na busca.

A prospecção realizada através de um conjunto de patentes similares, extraído de diversos bancos de patentes especializados em propriedade industrial pelo mundo, apontou para grandes investimentos em depósito de patentes em países mais desenvolvidos como Alemanha, Estados Unidos e França. Já em países como Brasil e Argentina, os resultados apresentaram impactos inexpressivos, o que mostra a deficiência de países em desenvolvimento para investir em tecnologia de ponta.

As engenharias elétrica, química e mecânica são as que mais investem estudos na área de reatores químicos, sendo que o setor químico é o que mais representa a tecnologia de reatores químicos, destacando-se a engenharia química, com cerca de 50\% dos depósitos atribuídos.

\section{Referências}

BASF. Disponível em: <https:/www.basf.com/br/pt/company/aboutus/history/1990-2014.html>. Acesso em: 25 fev. 2018.

BAHLMANN, C. Help Octimine, set. 2018. Disponível em: <https://www.octimine.com/help/> . Acesso em: 25 fev. 2018.

CRUZ, P.C.F.D. Aplicação de um novo reator de fluxo oscilatório no controlo da qualidade e tamanho dos cristais de paracetamol. 2015, 68 f. Tese (Mestrado Integrado em Engenharia Química) -LEPABE - Laboratório de Engenharia de Processos, Ambiente, Biotecnologia e Energia, Faculdade de Engenharia da Universidade do Porto, Porto, 2015.

GOIS, V. M. J.; OLIVEIRA, D. S.; CHAGAS, A. R. R.; LOPES C. F. D.; ANNA, S. S. C. M.; SILVIA F. G. Avaliação de tecnologias em reatores para produção de biodiesel a partir da análise dos pedidos de patentes. Cadernos de prospecção, São Cristovão, RJ, n. 4, p. 205-213, 2012.

KLEINA, N. Disponível em: < https:/www.tecmundo.com.br/lg/117567-historia-lg-rainhas-da-coreiasul.htm >. 13 jun. 2017. Acesso em: 25 fev. 2018.

LONZA Ltd. Dominique Roberge; Norbert Kockmann; Michael Gottsponer; Markus

Eyholzer. Method of manufacturing a reactor and set of reactors. EP2429695A1, 12 mai. 2010;

21 mar. 2012. 
MAYERHOFF, Z. D. V. Uma Análise Sobre os Estudos de Prospecção Tecnológica. Cadernos de Prospecção, Rio de Janeiro, n. 1, p. 7-9, 2008.

SATURNINO, W. L. S. Prospecção Tecnológica de Reatores Químicos. 2018. Trabalho de Conclusão de Curso (Graduação), Universidade Federal de Campina Grande, Campina Grande, fev. 2018.

SPEZIALI, G. M., SINISTERRA, D. R. Buscas de informações tecnológicas com base em dados de patentes: estudo de caso dos líquidos iônicos no brasil. Quim. Nova, Belo Horizonte, n. 8, p. 1.1321.138, nov./mar. 2012.

SIEMENS AG. Werner Dipl Ing Meier, Walter Dipl Ing Sauermann. Nuclear reactor element, in particular control. DE4041348A1, 21 dez. 1990; 25 jun. 1992.

VODAFONE PILOTENTWICKLUNG GMBH. Armin Dr. BartschGünter Dr. Hausinger. Reaktor. EP1224967A2, 15 jan. 2002, 24 jul. 2002.

\section{Sobre os autores}

\section{Wilton Lima Silva Saturnino}

E-mail: wiltonsaturnino@gmail.com

Graduado em Engenharia Química pela Universidade Federal de Campina Grande (2018).

Endereço Profissional: UFPB - Rua Aprígio Veloso, 882. Bairro Universitário - CEP: 58429-140 - Campina Grande - PB.

\section{Sidinei Kleber Silva}

E-mail: sidinei@eq.ufcg.edu.br

Graduado, Mestre (2006-2008) e Doutor (2009-2013) em Engenharia Química pela Universidade Federal de Campina Grande (UFCG).

Endereço Profissional: UFPB - Av. Aprígio Veloso 882 - C. Universitária, Bodocongó, CEP: 58109-970 - Campina Grande, PB. 